\title{
Disruption of natural grooming chains after striatopallidal lesions
}

\author{
KENT C. BERRIDGE \\ University of Michigan, Ann Arbor, Michigan \\ and \\ JOHN C. FENTRESS \\ Dalhousie University, Halifax, Nova Scotia, Canada
}

\begin{abstract}
Actions, like words, must be ordered into coordinated sequences. Action "syntax rules" exist for natural behavior such as gooming in rats. One rule produces a highly stereotyped chain of grooming actions. We report that the loss of intrinsic neurons from the corpus striatum, after injections of neurotoxic kainic acid, disrupts execution of this syntactic chain while preserving component actions. Striatopallidal circuitry may contribute to the effective serial organization even of natural action into certain centrally generated sequential patterns.
\end{abstract}

Since Karl Lashley (1951) first pointed out the ubiquity of the problem of "action syntax," the question of how distinct actions are organized by the brain into useful, coordinated sequences has received increasing attention. The control of the sequential structure of behavior has been approached primarily through the use of serial learning tasks, in which a person or animal must memorize a sequence of events and recall the order later. Experiments using primarily these tasks have identified the striatopallidal complex and the frontal cortex as particularly important to performance that requires serial ordering (e.g., Brunner, Kornhuber, Seemüller, Suger, \& Wallesch, 1982; Cools, 1985; Deecke, Kornhuber, Lang, \& Schreiber, 1985; Divac, 1972; Iversen, 1973, 1979; Jacobsen, 1936; Jaspers, Schwarz, Sontag, \& Cools, 1984; Kolb, 1984; Kolb \& Whishaw, 1985; Mogensen \& Divac, 1984; Rosvold, Mishkin, \& Szwarcbart, 1958; Van den Bercken \& Cools, 1982).

Serial learning is undoubtedly crucial to behavior. However, the nearly exclusive use of tasks requiring learning leaves open the question of whether the neural mechanisms involved are restricted to sequential memory and to the learning of order or whether they serve a more global sequencing role for all behavior, organizing even streams of untrained action into structured patterns in the way denoted by Lashley's term, "action syntax."

Sequential analyses of behavior that does not depend on training can help answer this question. In this way, for example, Kolb, Whishaw, and Schallert (1977) showed that orbital frontal cortex lesions disrupted natural

This work was supported by grants from the National Institutes of Health (NS23959) and The University of Michigan (Rackham faculty) to Kent C. Berridge and from the Canadian Natural Sciences and Engineering Research Council (A9787) and Medical Research Council (MS76609) to John C. Fentress. Requests for reprints should be sent to Kent C. Berridge, Department of Psychology, The University of Michigan, Neuroscience Laboratory Building, Ann Arbor, MI 48109. grooming sequences in rats, to the extent that grooming bouts were shortened and the distribution of behavioral components was affected (see also Schmidt, 1984). The shortening of bouts led Kolb et al. (1977) to suggest a global "inability to put together (that is, to sequence) long behavioral chains."

Recently, we described a number of objectively identifiable rules that organize the sequential structure of grooming and feeding actions in rats (Berridge, Fentress, \& Parr, 1987). One of the strongest patterning rules consists of the syntactic chaining of up to 25 actions in predictable order, a rule that can be seen in the facial grooming of virtually every rat. This stereotyped sequence appears to be initiated by a "trigger" sensitive to the spatial and temporal configuration of ongoing grooming strokes (Berridge et al., 1987). It begins with a bilateral bout of 5-9 rapid elliptical forepaw strokes $(6-7 \mathrm{~Hz})$ over the mystacial vibrissae, each stroke bilaterally symmetrical. The bout of elliptical strokes is followed immediately by a short series of slower, unilateral or asymmetrical strokes (1-4) of small to intermediate amplitude and then by a set of bilateral strokes (3-8) of large amplitude, followed by tucking of the head. Finally, the chain is terminated by a bout of body licking (Figure 1). This chain occurs with a sequential reliability more than 13,000 times greater than could be expected by chance based upon component action probabilities (Berridge et al., 1987).

Syntactic chains also have identifying features in addition to their sequential structure. One feature is that the control of the form of grooming actions becomes less dependent on facial trigeminal sensory input, and appears to revert to a more centrally programmed mode, for the duration of the chain. The distortions of grooming action form produced by trigeminal deafferentation thus tend not to invade actions within a chain (Berridge \& Fentress, 1986). Conceivably, the suppression of sensorimotor responsiveness during syntactic chaining might not only 

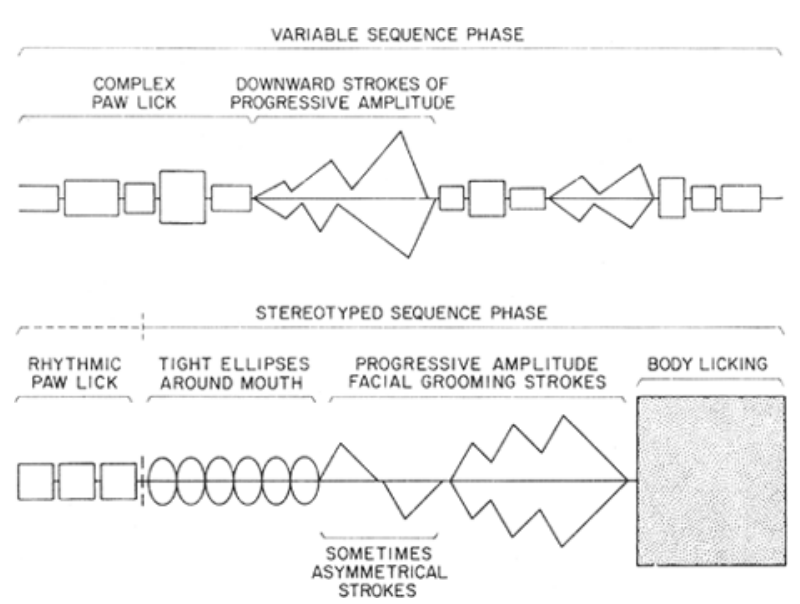

Figure 1. Schematic representation of facial grooming. A sequentially variable phase is shown in the top panel; the syntactic chain is shown in the bottom panel. Open squares denote paw licking, filled square denotes body licking, and ellipses denote elliptical forepaw strokes over the mystacial vibrissae. Diagonal deviations of the lines above and below the horizontal axis denote movements of the two forepaws upward along the face away from the nose. Approximately 5 sec of grooming is represented in each phase.

coincide with the stereotyped sequential structure but actually contribute to it. A suppression of trigeminal sensory processing might reduce somatosensory distractions that would otherwise compete with the centrally generated chain program for action-sequence control. The reversion of action form to a more centrally programmed mode would follow as a necessary consequence.

The corpus striatum complex (caudate-putamen and globus pallidus) is a likely neural candidate for participation in the control of both sensorimotor modulation and global behavioral sequencing (e.g., Evarts, Kimura, Wurtz, \& Mikosaka, 1984; Evered \& O'Conner, 1984; Jaspers et al., 1984; Marsden, 1980). A number of investigators have proposed that circuitry within the corpus striatum may systematically enhance and suppress sensorimotor loops and internal programs in ways that are appropriate to specific behavioral contexts (Abercrombie \& Jacobs, 1985; Cools, 1985; Iversen, 1979; Lidsky, Manetto, \& Schneider, 1985; Sabol, Neil, Wages, Church, \& Justice, 1985 ), thus programming the "ordering and sequencing of behavioral states" (Cools, 1985). This striatal role could be important to the performance of syntactic grooming chains. Multiunit bursts of activity occur in globus pallidus neurons during grooming (McKenzie, Everett, \& Kunze, 1984). Electrical or chemical stimulation of the neostriatum (caudate-putamen) or of the nigrostriatal projection can alter the responsiveness of trigeminal motor neurons to trigeminal sensory stimulation (Huston, Morgan, \& Steiner, 1987; Labuszewski \& Lidsky, 1979). Conversely, lesions of the neostriatum and globus pallidus produce a facilitation of trigeminal sensorimotor responsiveness (Schneider, 1987a; Schneider, Denaro, \& Lidsky, 1982).
In the present experiment, we sought to determine whether striatal neural circuity made an important contribution in organizing natural grooming actions into syntactic chains. To do this, we administered intrastriatal injections of kainic acid to induce damage to intrinsic neurons of the corpus striatum, and then observed the rats to see if their execution of grooming chains had been impaired. In addition, to examine explicitly the relative roles of central pattern generation versus peripheral reflex or feedback mechanisms in guiding behavioral sequence, we compared our striatal-lesion data with previously collected data on the effect of loss of tactile sensory cues and feedback from the face (induced by trigeminal deafferentation).

\section{METHOD}

Forty-one male Sprague-Dawley rats weighing 300-400 g were housed in pairs on a 14:10 light:dark cycle (light onset 8:00 a.m.). The rats were divided into an anterior striatal lesion group (10 rats), a posterior striatal lesion group (10 rats), a striatal vehicle control group ( 5 anterior rats; 5 posterior rats), and an intact control group (11 rats). In addition, to allow for a direct comparison of the effects on chaining of central striatal lesions versus peripheral sensory loss, syntactic chaining data from 9 rats from a previous trigeminal deafferentation study (Berridge \& Fentress, 1987) were included in the quantitative analysis.

\section{Surgery}

The rats were anesthetized with Ketamine $\mathrm{HCl}(100 \mathrm{mg} / \mathrm{kg})$ and acepromazine (1 mg/kg) prior to surgery. Kainic acid (1 $\mu \mathrm{g}$ in $0.5 \mu \mathrm{l}$ phosphate buffer) was injected at each striatopallidal site over a 5min period; the needle was kept in place another $10 \mathrm{~min}$. Diazepam $(8 \mathrm{mg} / \mathrm{kg})$ was given before and after kainic acid injections to control convulsant activity. Anterior striatal coordinates were $1.7 \mathrm{~mm}$ anterior to bregma, $\pm 2.2 \mathrm{~mm}$ lateral from midline, and $5.5 \mathrm{~mm}$ below skull surface. Posterior striatopallidal coordinates were -0.8 , $\pm 3.5,-6.5$, using the same landmarks. Control-vehicle injections were of phosphate buffer alone. Trigeminal deafferentation was performed by selective bilateral transection of the inferior alveolar, lingual, and auriculotemporal nerves of the mandibular trigeminal branch and of the anterior superior alveolar and infraorbital nerves of the maxillary trigeminal branch. Deafferentation was performed under visual guidance with the aid of a dissecting microscope.

After surgery, the rats were maintained on commercial baby cereal mixed with water to form a loose mash, in addition to chow pellets. Aphagic rats received three daily intragastric tube feedings of $12 \mathrm{ml}$ of a vitamin-supplemented liquid diet (sweetened condensed milk with water).

\section{Behavioral Testing}

Natural grooming sequences were videotaped between 2:00 and 6:00 p.m. during the 2 nd and 3 rd weeks after surgery. The rats were lightly sprayed with a water mist to induce grooming, and placed on a transparent plastic floor, under which a mirror was positioned to give the camera a clear close-up view of each rat's head and upper body. At least $10 \mathrm{~min}$ of grooming was videotaped for each rat. Videotapes were analyzed in slow motion (frame by frame to $1 / 10$ actual speed) by trained observers blind to the experimental condition of each rat, for the occurrence and completion of stereotyped chain sequences. Briefly, the criterion for successful completion of a chain was that the initiating bout of elliptical strokes be followed immediately by at least two downward unilateral or bilateral strokes of larger amplitude, and then by flank licking within $5 \mathrm{sec}$. Any actions other than downward unilateral or bilateral fore- 
paw strokes over the face that intervened between the ellipitical stroke bout and body licking were considered "extra" intrusions. The graphic grooming notation system depicted in Berridge and Fentress (1986) was used to record each chain.

\section{Histology}

At the conclusion of the experiment, each rat was deeply anesthetized and perfused. Brains were frozen, sectioned, mounted on slides, and stained with cresyl violet. Sections were microscopically examined to assess gliosis and the loss of neuron cell bodies.

\section{RESULTS}

\section{Lesion Histology}

Kainic acid produced marked gliosis and neuron loss in the nucleus caudate-putamen (neostriatum) and globus pallidus. Cell loss in the globus pallidus and nucleus basalis was much more extensive in the posterior-injection group. Typical and maximal extents of kainic acid lesions are shown in Figure 2. Marked cell loss could also be seen in the anterior lateral thalamic nuclei in 2 of the posterior-injection rats, and in the lateral septum, dorsal medial thalamus, neocortex, anterior hypothalamus, and amygdala in 1 to 3 of the posterior-injection rats.

\section{Behavioral Chaining}

In normal intact rats (Figure 3), the appearance of the chain-initiating bout (of 5 to 9 elliptical strokes at 6-7 Hz) was followed by successful completion of the rest of the sequence in over $90 \%$ of all cases. Chain completion did not differ between intact, vehicle control, and trigeminal deafferented groups by analysis of variance $[F(2,29)=$ $0.7]$, but vehicle controls were marginally lower than intacts by two-tailed $t$ test $(p<.05)$ (striatal injections of the buffer vehicle may create a small amount of nonspecific damage). Removal of somatosensory cues from the face by deafferentation did not reduce the percentage of chains carried to completion although it did increase the proportion of chains incorporating "extra" intrusions such as a forelimb flail or pause ( $p<.025, t$ test). Most importantly, however, kainic acid lesions of the corpus striatum resulted reliably in a significant disruption of chain completion (Figure 3). Both posterior- and anteriorlesion groups completed a smaller percentage of grooming chains than did the vehicle control group ( $t$ tests, $p<.01)$, the intact group $(p<.01)$, or the deafferented group $(p<.01)$. This disruption of syntax after corpus striatum lesions appeared to be one of rule completion and was not necessarily accompanied by a reduction in the number of chains that were begun. Kainic acid striatopallidal lesions reduced the rate of successful chain completion by at least $60 \%$ for anterior- and posterior-lesion groups. The number of chains begun, on the other hand, was lowered only for the posterior group, whose rate was lower than that of every other group (Mann-Whitney U,
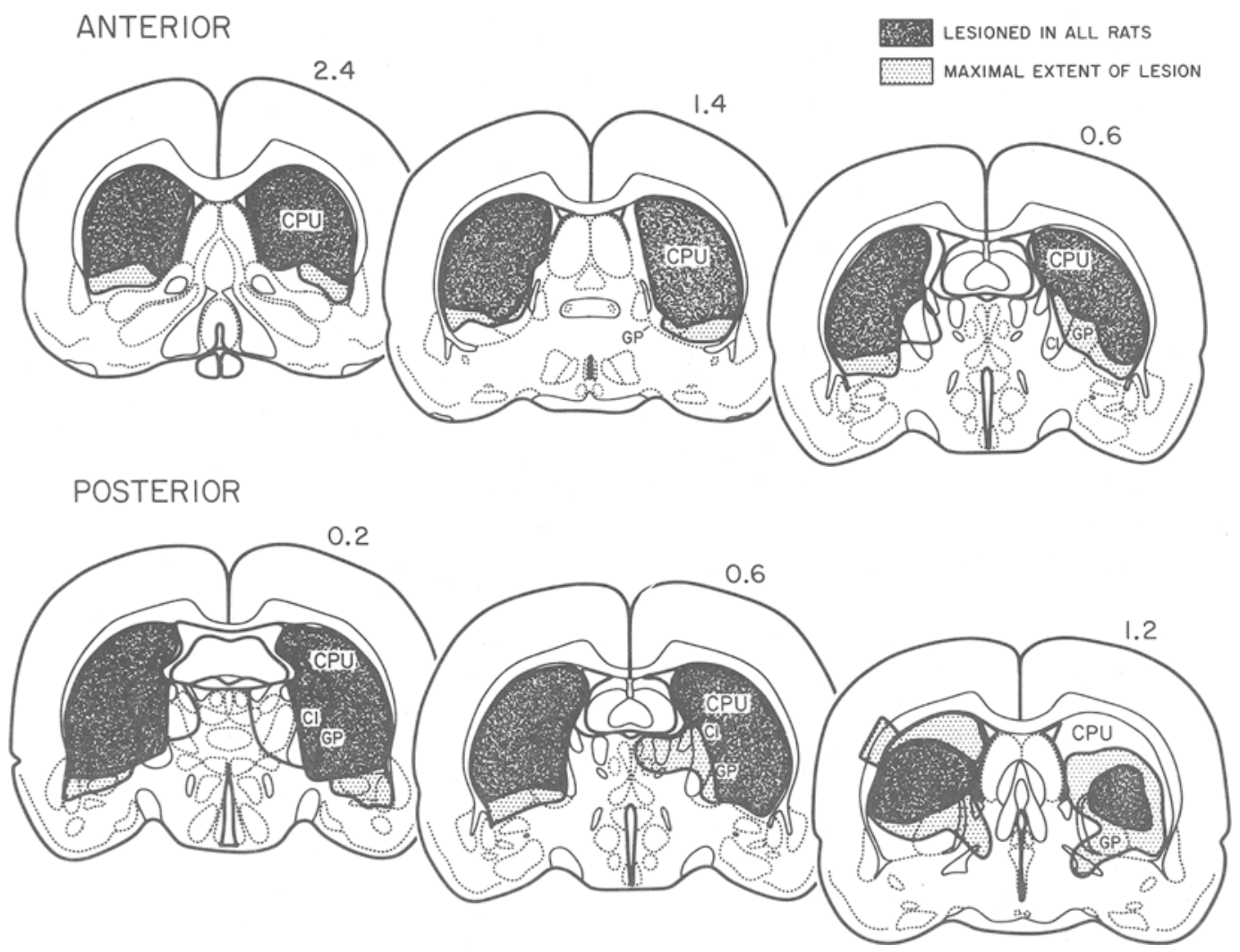

Figure 2. Extent of kainic-acid-induced lesions. Gray portions depict areas of neuronal cell body loss and gliosis shared by all members of a lesion group. Dotted portions depict maximal extents of individual lesions. 


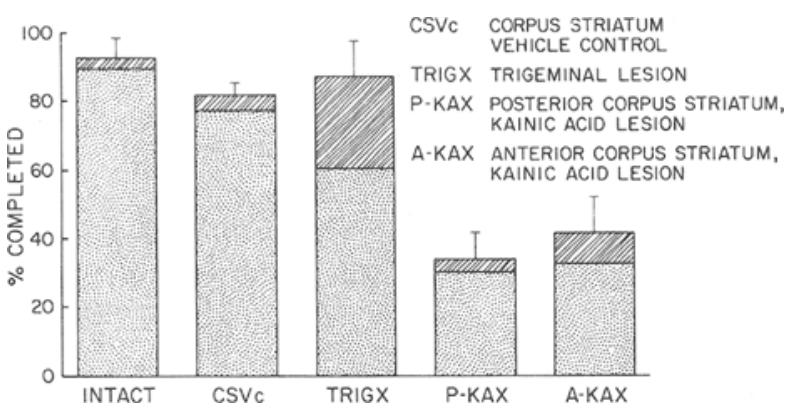

Figure 3. Percentages of syntactic chains that, once begun, were completed by intact, vehicle-injected, posterior striatal lesioned, and anterior striatal lesioned rats (mean \pm standard error). Dotted portions denote perfect syntax completion; striped portions denote successful completion with at least one intrusion by an "extra" action.

$p<.02)$. Anterior striatal lesions had no effect on the number of chains begun.

Notated examples of actual chains from the striatopallidal lesion and vehicle control groups are shown in Figure 4. Clearly, every component of the chain can be produced by rats with striatopallidal lesions. It can also be seen that the initial portion of chains produced by rats with striatal lesions was not markedly different from the initial portion of the control chains. But as the chain progressed, rats with striatopallidal damage had a greater probability of transgressing the stereotyped rule that leads to chain termination, and instead continued with an irregular series of grooming strokes and paw licks. Most instances of incomplete chains continued into extended face washing $(86 \%)$.

\section{DISCUSSION}

The disruption of syntactic chaining of grooming actions after central striatopallidal but not after peripheral trigeminal lesions provides support for the hypothesis that striatal circuitry helps achieve sequential patterns of untrained, as well as remembered, actions and events. The ability of both anterior and posterior striatopallidal lesions to disrupt chain completion may suggest either that an intermediate region overlapped by the two sites is responsible or that the relevant circuitry is anatomically distributed in both rostral and caudal portions of the heterogeneous corpus striatum. Unlike chaining, other consequences of these striatopallidal lesions, such as evoked hyperkinesia or aphagia, are not altered equally by these anterior and posterior lesions (Berridge, Treit, \& Fentress, in press).

Precisely why should the sequential organization of species-typical behavior be impaired by striatopallidal damage? There are a number of possible alternatives. Examination of notated chains reveals that rats with striatal damage are well able to execute the bout of initiating ellipses, to follow appropriately with the occasional smallamplitude unilateral or asymmetrical stroke, and to ascend to the beginning of the large-amplitude bilateral series. It is within this series of penultimate large-amplitude bilateral strokes that they become lost, however, and remain or return to rhythmic paw licks rather than go appropriately to body licking. It may be informative to note that these large strokes tend to be the most complex and variable in form of all the chain components.

A conclusion that rats with striatopallidal damage lack the capacity to perform body licking, the terminating action, is probably unwarranted, as the two completed chains in Figure 4 by rats with lesions suggest. We did not find a significant reduction in body-licking incidence as a whole in the lesion groups. Yet this transition from large bilateral strokes to body licking appears especially vulnerable.

A second alternative to account for this vulnerability would be to note that a major shift in body posture is required to switch from facial stroking to body licking, and to suppose that postural deficits produced by corpus striatum lesions make this transition impossible. Arguing against the sufficiency of a postural deficit as a full explanation of chaining failures, however, are the facts that (1) postural deficits are produced primarily by posterior lesions (Labuszewski, Lockwood, McManus, Edelstein, \& Lidsky, 1981; and confirmed by our own observations) whereas syntactic chaining is impaired by both anterior and posterior lesions, and (2) most failures to complete chains did not end in abortive attempts to body-lick or in visible loss of balance, but rather continued on without interruption into sequentially flexible facial grooming.

The answer to why rats with striatopallidal damage fail to complete syntactic grooming chains is likely to be more complicated than either of the possibilities given above. Two classes of answer appear most plausible. First, it is possible that striatal circuitry plays a role in the actual specification of action syntax, that is, in the central generation of the neuronal patterns that produce rule-governed sequences (e.g., Evarts et al., 1984; Jaspers et al., 1984; Kornhuber, 1974; Marsden, 1980). This possibility would account for chaining failure after a striatopallidal lesion by supposing that the syntactic rule itself can no longer be generated properly without the intrinsic striatopallidal neurons that constitute the central pattern generator (see Doty, 1976).

The other possible class of explanation focuses upon rule implementation rather than generation, and relies upon the intimate association between striatopallidal motor control and sensory function mentioned earlier (see Schneider \& Lidsky, 1987). This line of explanation would suggest that the corpus striatum contributes to behavioral sequencing not by generating the syntax rules themselves, but by modulating sensorimotor function. Sensorimotor modulation, performed phasically and often, could rapidly switch control between external guiding stimuli and centrally generated rules in order to guide the sequence of action appropriately (e.g., Abercrombie \& Jacobs, 1985; Cools, 1985; Huston, Nef, Papadopoulos, 
VEHICLE-INJECTED CONTROL COMPLETED CHAINS

TRIAL 8-2I
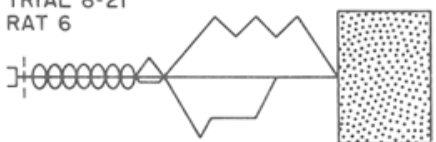

TRIAL 8-2।

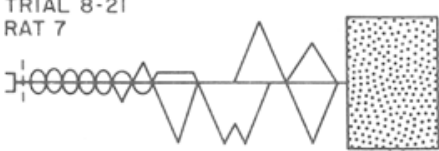

TRIAL ${ }^{10-25}$

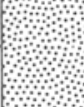

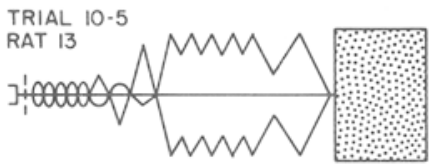

TRIAL $\|-\mid$

RAT 22

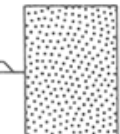

TRIAL $10-31$

RAT ${ }^{23}$

TRIAL II-25

TRIAL II-25

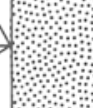

TRIAL ||-25

I'DAT 30

RAT 31 II-25
CORPUS STRIATUM LESIONS

ANTERIOR LESIONS - INCOMPLETE CHAINS

TRIAL 10-5

RAT 16

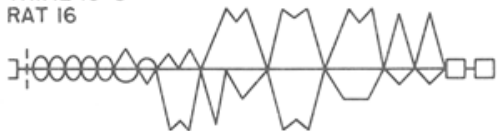

RIGHT
EAR
EYE
NOSE
NOSE
EYE
EAR
LEFT

TRIAL 10-31

RAT 24

iemonos

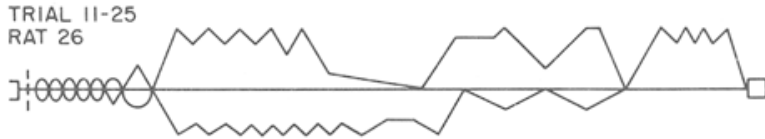

TRIAL II-26

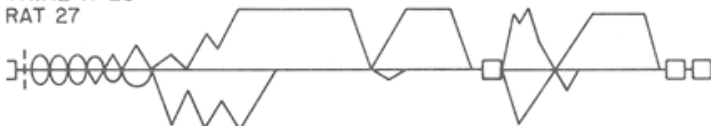

POSTERIOR LESION - INCOMPLETE CHAINS

TRIAL 8-27

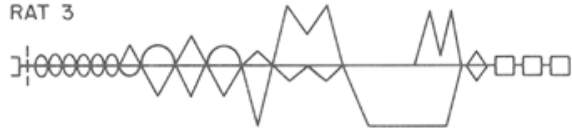

TRIAL 8-27

RAT 4

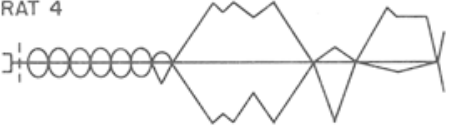

TRIAL IO-5

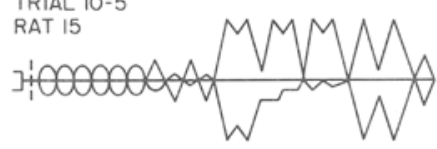

TRIAL I0-3|

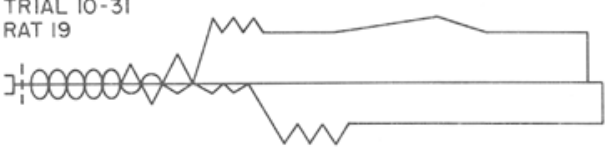

ANTERIOR LESION - COMPLETED CHAIN

TRIAL |1-25

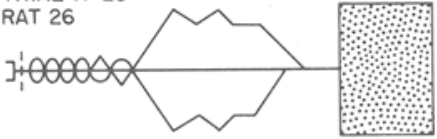

POSTERIOR LESION - COMPLETED CHAIN

TRIAL IO-5

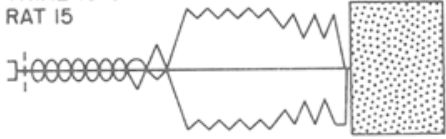

Figure 4. Examples of actual syntactic chains shown by vehicle-control (left panel) or striatal lesioned (right panel) rats. The symbols are as in Figure 1. 
\& Weizl, 1980; Iverson, 1979; Lidsky et al., 1985; Sabol et al., 1985; Schneider, 1987a, 1987b). The striatum is envisioned here as a hierarchically superior controller that can shift the control of action among potentially competing signals. A similar role has been implicated for cortical connections (Vanderwolf, Kolb, \& Cooley, 1978). Regarding syntactic grooming chains, a phasic suppression of trigeminal sensorimotor responsiveness has been shown by the fact that grooming actions occurring within the chain are protected from the deafferentation-induced distortions of form that characterize the same actions when they occur outside of the chain (Berridge \& Fentress, 1986). A disruption of sensorimotor modulation by a striatal lesion could leave the syntactic chain vulnerable to interruptions from competing tactile stimuli, even though the syntax-generating circuit itself might be intact. The pattern of syntactic failures relatively late in the chain, and the invasion by sequentially flexible (possibly stimulus-guided) facial grooming noted above are consistent with the hypothesis that centrally generated chain signals tend to lose in competition against distracting facial stimuli after striatopallidal lesions.

Answers to future empirical questions can help decide between these two theoretical possibilities. If striatopallidal circuits implement but do not generate syntactic chains, can basic chain generation be shown to be carried out elsewhere in the brain? If phasic sensorimotor modulation is crucial to striatal implementation of syntactic chaining, can modulation of sensorimotor thresholds be explicitly demonstrated during chaining and will striatopallidal lesions alter this? Whatever the answers to these questions, we can at least conclude from our current results that the syntactic structure even of natural and untrained behavioral sequences depends importantly upon striatopallidal function.

\section{REFERENCES}

ABERCRombiE, E. D., \& JACOBS, B. L. (1985). Dopaminergic modulation of sensory responses of striatal neurons: Single unit studies. Brain Research, 358, 27-33.

Berridge, K. C., \& Fentress, J. C. (1986). Contextual control of trigeminal sensorimotor function. Joumal of Neuroscience, 6, 325-330.

Berridge, K. C., \& Fentress, J. C. (1987). Deafferentation does not disrupt natural rules of action syntax. Behavioral Brain Research, 23, 69-76.

Berridge, K. C., Fentress, J. C., \& PARr, H. (1987). Natural syntax rules control action sequence of rats. Behavioral Brain Research, 23, 59-68.

Berridge, K. C., Treit, D., \& Fentress, J. C. (in press). A triggered hyperkinesia induced in rats by lesions of the corpus striatum. $E x$ perimental Neurology.

Brunner, R. J., Kornhuber, H. H., Seemüller, E., Suger, G., \& WALLESCH, C. W. (1982). Basal ganglia participation in language pathology. Brain \& Language, 16, 281-299.

Cools, A. R. (1985). Brain and behavior: Hierarchy of feedback systems and control of input. In P. P. G. Bateson \& P. H. Klopfer (Eds.), Perspectives in ethology (Vol. 6, pp. 109-168). New York: Plenum.

Deecke, L., Kornhuber, H. H., LANG, M., \& Schreiber, H. (1985) Timing function of the frontal cortex in sequential motor and learning tasks. Human Neurobiology, 4, 143-154.
Divac, I. (1972). Neostriatum and functions of prefrontal cortex. Acta Neurobiologiae Experimentalis, 32, 461-478.

Doty, R. W. (1976). The concept of neural centers. In J. C. Fentress (Ed.), Simpler networks and behavior (pp. 251-265). Sunderland, MA: Sinauer.

Evarts, E. V., Kimura, D., Wurtz, R. M., \& Mikosaka, O. (1984). Behavioral correlates of activity in basal ganglia neurons. Trends in Neuroscience, 7, 447-453.

Evered, D., \& O'ConNor, M. (Eds.) (1984). General discussion I. In Functions of the basal ganglia (CIBA Symposium 107, pp. 103-113). London: Pitman.

Huston, J. P., Morgan, S., \& Steiner, H. (1987). Plasticity in crossed efferents from the substantia nigra as related to behavioral reorganization. In J. S. Schneider \& T. I. Lidsky (Eds.), Basal ganglia and behavior: Sensory aspects of motor functioning (pp. 89-102). Lewiston, NY: Hans Huber

Huston, J. F., Nef, B., Papadopoulos, G., \& Weizl, H. (1980). Activation and lateralization of sensorimotor field for perioral biting reflex by intranigral GABA and by systemic apomorphine in the rat . Brain Research Bulletin, 5, 745-750.

IVERSEN, S. D. (1973). Brain lesions and memory in animals. In J. A. Deutsch (Ed.), The physiological basis of memory. New York: Academic.

IVERSEN, S. D. (1979). Behavior after neostriatal lesions in animals. In I. Divac \& R. G. E. Oberg (Eds.), The neostriatum (pp. 183-195). New York: Pergamon.

JACOBSEN, C. F. I. (1936). The functions of the frontal association areas in monkeys. Comparative Psychology Monographs, 13, 1-60.

Jaspers, R., Schwarz, M., Sontag, K.-H., \& CoOls, A. R. (1984). Caudate nucleus and programming behavior cats: Role of dopamine in switching motor patterns. Behavioral Brain Research, 14, 17-28.

KoLB, B. (1984). Functions of the frontal cortex of the rat: A comparative review. Brain Research Review, 8, 65-98.

KolB, B., \& WhishaW, I. Q. (1985). Fundamentals of human neuropsychology (2nd ed.). New York: W. H.Freeman.

Kolb, B., Whishaw, I. Q., \& Schallert, T. (1977). Aphagia, behavior sequencing and body weight set point following orbital frontal lesions in the rat. Physiology \& Behavior, 19, 93-103.

KoRNHUBER, H. H. (1974). Cerebral cortex, cerebellum, and basal ganglia: An introduction to motor functions. In F. O. Schmitt \& F. G. Worden (Eds.), The neurosciences, Third Study Program (pp. 267280). Cambridge, MA: MIT Press.

LABUSZEWSKI, T., \& LIDSKY, T. I. (1979). Effects of basal ganglia stimulation on brainstem trigeminal neurons. Experimental Neurology, 65 , 471-477.

Labuszewski, T., Lockwood, R., McManus, F. E., Edelstein, L. R., \& LIDSKY, T. I. (1981). Role of postural deficits in oro-ingestive problems caused by globus pallidus lesions. Experimental Neurology, 74, 93-110.

LASHLEY, K. S. (1951). The problem of serial order in behavior. In L. A. Jeffress (Ed.), Cerebral mechanisms in behavior (pp. 112-156). New York: Wiley.

Lidsky, T. I., Manetto, C., \& Schneider, J. S. (1985). Consideration of sensory factors involved in motor functions of the basal ganglia. Brain Research Reviews, 9, 133-146.

Marsden, C. D. (1980). The enigma of the basal ganglia and movement. Trends in Neuroscience, 3, 284-287.

McKenzie, J. S., EveretT, P. W., \& Kunze, W. A. (1984). Multiunit bursts in rat pallidum during grooming and stereotyped jaw movements. Brain Research Bulletin, 13, 493-496.

Mogensen, J., \& Divac, I. (1984). Sequential behavior after modified prefrontal lesions in the rat. Physiological Psychology, 12, 41-44.

Rosvold, H. H., Mishkin, M., \& Szwarcbart, M. D. (1958). Effect of subcortical lesions in monkey on visual discrimination and single alternation performance. Journal of Comparative \& Physiological Psychology, 51, 437-444.

Sabol, K. E., Neill, D. B., Wages, S. A., Church, W. H., \& Justice, J. B. (1985). Dopamine depletion in a striatal subregion disrupts performance of a skilled motor task in rats. Brain Research, 335, 33-43. 
SCHmIDT, W. J. (1984). L-Dopa and apomorphine disnupt long but not short behavioral chains. Physiology \& Behavior, 33, 671-680.

SCHNEIDER, J. S. (1987a). Basal ganglia-motor influences: Role of sensory gating. In J. S. Schneider \& T. I. Lidsky (Eds.), Basal ganglia and behavior: Sensory aspects of motor functioning (pp. 103-121). New York: Hans Huber.

SCHNEIDER, J. S. (1987b). Ingestion-related activity of caudate and entopeduncular neurons in the cat. Experimental Neurology, 95, 216-223.

SchneIder, J. S., Denaro, F. J., \& LidSky, T. I. (1982). Basal ganglia: Motor influences mediated by sensory interactions. Experimental Neurology, 77, 534-543.

SCHNEIDER, J. S., \& LIDSKY, T. I. (Eds.) (1987). Basal ganglia and behavior: Sensory aspects of motor functioning. New York: Hans Huber.

VAN DEN Bercken, J. H. L., \& CoOls, A. R. (1982). Evidence for the role of the caudate nucleus in the sequential organization of behavior. Behavioral Brain Research, 4, 319-337.

Vanderwolf, C. H., Kolb, B., \& Cooley, R. K. (1978). Behavior of the rat after removal of the neocortex and hippocampal formation. Joumal of Comparative \& Physiological Psychology, 92, 156-175.

(Manuscript received July 30, 1987;

revision accepted for publication November 10, 1987.) 